\section{Shwachman Diamond Syndrome: an emergency challenge}

PAOLA COCCIA • ANTONIO RUGGIERO • GIORGIO ATTINÀ • PALMA MAURIZI ILARIA LAZZARESCHI • FRANCESCO MOLINARI • RICCARDO RICCARDI

\author{
ANTONIO RUGGIERO ( $\square) \bullet$ \\ GIORGIO ATTINÀ • PALMA MAURIZI • \\ ILARIA LAZZARESCHI • PAOLA COCCIA • \\ RICCARDO RICCARDI \\ Division of Paediatric Oncology. \\ Pol. A. Gemelli \\ Catholic University of Rome \\ Largo A. Gemelli 8 \\ 00168 Roma, Italy \\ Phone: +39063058203 \\ Fax: +39063052751 \\ E-mail: ruggiero@rm.unicatt.it \\ FRANCESCO MOLINARI \\ Department of Radiological Sciences, \\ Catholic University of Rome, Rome, Italy
}

\begin{abstract}
Shwachman Diamond Syndrome is a rare congenital disorder characterized by pancreatic insufficiency, bone marrow dysfunction and skeletal abnormalities. No specific test is available for a definitive diagnosis for SDS. In the presence of clinical features of SDS, pancreatic insufficiency should be tested for or lipomatosis radiologically demonstrated. New directions might be found in genetic analysis. Sometimes, a life-threatening event may be the first clinical manifestation of the syndrome.
\end{abstract}

Keywords: lipomatosis, SDS, diagnosis, children

\section{Shwachman Diamond Syndrome: a diagnostic challenge.}

Shwachman Diamond syndrome (SDS) is a congenital disorder, first described in 1964 in five patients showing exocrine pancreatic insufficiency and leucopenia. (1)

Although SDS is a rare disorder, it is the most common cause of pancreatic insufficiency in children next to cystic fibrosis and probably the third most common inherited bone marrow failure syndrome after Fanconi's anemia and Diamond-Blackfan anemia.

An autosomal recessive mode of inheritance has been described.

Up to December 2000, more than 300 cases of SDS had been reported in the literature. (2)

\section{Clinical features}

SDS usually manifests itself in infancy with multisystemic abnormalities including exocrine pancreatic insufficiency, hematological and skeletal abnormalities. The clinical phenotype of SDS is extremely heterogeneous. $(3,4)$ No unifying pathogenetic mechanism has yet been shown to be responsible for SDS, although new insights into molecular, genetic and cellular basis of this rare disease have recently been described. (3)

Pancreatic insufficiency. Histological analysis of the pancreas shows preserved ducts and paucity of acini with fatty replacement in the gland. More than $98 \%$ of the exocrine acinar capacity of the pancreas must be lost before the clinical symptoms of pancreatic insufficiency appear. Steatorrhea is noted in the first 6 months of life in about $50 \%$ of affected children and increases to $90 \%$ by the age of one. (5) Steatorrhea and malabsorption might lead to malnutrition and fat-soluble vitamin deficiency (A, D, E, K). (4) Hematological abnormalities. Inter- mittent neutropenia is the most common hematological finding in SDS, occurring in $88-100 \%$ of patients and it can be persistently or intermittently severe, with a neutrophil count of $<0.5 \times 10^{9} / \mathrm{L}$. (6) The neutrophils in patients affected with SDS may have impaired mobility and defects in migration or chemotaxis. (5) Alterations in neutrophil cytoskeletal/ microtubular integrity and function may play a significant role in causing the defective chemotaxis.

Therefore, more of the bone marrow cellular elements can be involved. Hematological manifestations other than neutropenia include anemia, raised fetal hemoglobin levels, thrombocytopenia and aplastic anemia. Anemia is the second most common cytopenia in SDS, recorded in up to $80 \%$ of patients. It is usually mild and normochromicnormocytic with a low reticulocyte count. (2) Fetal hemoglobin was increased in $80 \%$ of patients. (7)

Many patients develop a mild and clinically insignificant thrombocyto- 
penia, defined by a platelet count $<$ $150 \times 10^{9} / \mathrm{L}$.

Pancytopenia occurs in $10-65 \%$ of patients. Both anemia and thrombocytopenia are usually mild to moderate in these cases but neutropenia tends to be severe. Trilineage cytopenia is associated with a poor prognosis, that is, a higher probability of developing symptomatic severe aplasia. (8) Hemopoiesis is probably affected by defects in the marrow-stromal support structure. (2)

As with other constitutional bone marrowfailure syndromes, retrospective studies suggested a high propensity for malignant transformation into myelodysplastic syndromes and acute myeloid leukemia (25-33\% of the patients). The detection of cytogenetic abnormalities in the bone marrow is a marker of clonal evolution and malignant transformation. (2) The isochromosome $7 q$ (i7q) was found with malignant myeloid transformation in $25 \%$ of SDS patients. $(2,9,10)$

Skeletal abnormalities. The musculoskeletal system is the most commonly affected organ after the hematopoietic and pancreatic systems; more than half of SDS patients have metaphyseal dysostoses, which usually involves the femoral head. Radiographs may detect these alterations after 12 months of age. Approximately $30-50 \%$ of patients show thoracic cage defects, including shortened ribs with flared ends and chostocondral thickening. (11) Other skeletal problems include syndactyly, clinodactyly, supernumerary thumbs, kyphosis and scoliosis. (12) Significant osteoporosis and osteomalacia are often observed, most likely due to a decrease in vitamin $\mathrm{D}$ and vitamin $\mathrm{K}$ absorption, which result in a high risk of pathological fractures. (11)

Immune dysfunction. Patients with SDS experience recurrent viral, bacterial and fungal infections, including otitis media, bronchopneumonia, osteomyelitis, skin infection and septicemia. The quantitative and qualitative defects in SDS neutrophils contribute to these infections. Various defects in B and $\mathrm{T}$ cells have been reported, both quanti- tative (low lgG levels, low percentage of circulating B lymphocytes) and qualitative (decreased in vitro lymphocyte proliferation, lack of specific antibody production). (13)

Other involvement. Failure to thrive is commonly observed in patients affected with SDS and various factors may contribute to this condition, including malabsorption, recurrent infections and metaphyseal dysostoses. Birth weight is often normal, but more than $50 \%$ of patients are still below the $3^{\text {rd }}$ percentile for height when they are one year old. (8) All patients exhibit delayed bone age and delayed puberty. Increased risk of caries, mucositis and periodontal infections, dental dysplasia and delayed dentition of permanent teeth are frequently seen. (14)

Hepatomegaly and raised liver enzymes are observed in 50-75\%, especially in young children. (2)

Severe eczema is often seen at presentation. (15)

Recent studies suggest that cognitive and attention difficulties are experienced by a large number of affected children. (16)

\section{Diagnosis}

The clinical phenotype of SDS is extremely heterogeneous showing a wide range of abnormalities and symptoms. At present, no specific biochemical or genetic test is available for a definitive diagnosis for SDS. Based on current knowledge of the SDS phenotype, the diagnosis requires evidence of exocrine pancreatic dysfunction and characteristic hematological abnormalities. Short stature, skeletal abnormalities, hepatomegaly or biochemical abnormalities of the liver are supportive findings of the diagnosis. Clinical diagnostic criteria used by Dror and Freedman are listed in table 1. (2) Therefore, SDS must be suspected when neutropenia and recurrent infections are present, inall children with delayed growth, short stature, skeletal abnormalities or steatorrhea.

The recognition of pancreatic dysfunction has been regarded as an essential part of the diagnosis of SDS. Typically, exocrine enzyme secretion is diminished. The function of the exocrine pancreas is difficult to assess because of its anatomical inaccessibility and large reserve capacity. The "gold standard", the direct pancreatic stimulation test provides quantitative and accurate measures of pancreatic function but is not widely used in clinical practice because of its complexity, cost and invasiveness. Pancreatic insufficiency is assessed by the absence or decrease of trypsin, lipase and amylase activity in pancreatic fluid, sampled through a nasogastric tube, from less than $2 \%$ to approximately $10-14 \%$ of the reference range after a quantitative stimulation

\section{Table 1: Diagnostic criteria for SDS.}

Exocrine pancreatic dysfunction (at least one of the following):

- Abnormal quantitative pancreatic stimulation test.

- Serum cationic trypsinogen below the normal range.

- Abnormal 72 hour fecal fat analysis plus evidence of pancreatic lipomatosis by ultrasonographic examination or CT scan.

AND Hematological abnormalities (at least one of the following):

- Chronic (on two occasions at least 6 weeks apart) single lineage or multilineage cytopenia with bone marrow findings consistent with a productive defect:

- neutrophil $<1.5 \times 10^{9} / 1$

- hemoglobin concentration <2 standard deviations below mean, adjusted for age

- thrombocytopenia $<150 \times 10^{9} / /$.

-Myelodysplastic syndrome. 
test with intravenous secretin and cholecystokinin. (2)

In SDS, low serum immunoreactive trypsinogen values are based on reduced acinar enzyme production, because pancreatic failure is due to hypoplasia. On the other hand, in cystic fibrosis, the serum immunoreactive trypsinogen level is high because of the underlying pathophysiological features affecting the pancreatic duct and the inability to secrete digestive enzymes into the duodenal lumen.

In some patients with SDS, the exocrine pancreatic enzyme production is so low that the patient clinically manifests with steatorrhea and has abnormal 72-hour fecal fat studies. However, the absence of steatorrhea does not exclude the diagnosis of SDS. (8)

Syndromes that can manifest pancreatic pathology include: Beckwith Wiedemann syndrome, von HippelLindau disease, autosomal dominant polycystic kidney disease and Pearson disease (pancreatic insufficiency, neutropenia, marrow ring sideroblasts and vacuolated erythroid and myeloid precursors). Pancreatic abnormalities, associated with fatty replacement of the gland, can be found also in cystic fibrosis, Cushing syndrome, obesity or Johanson Blizzard Syndrome. (17)

A negative sweat test allows us to rule out the diagnosis of cystic fibrosis.

Histological analysis of the pancreas shows preserved ducts and paucity of acinis with fatty replacement in the gland, a feature that may be delineated by appropriate radiological imaging studies.

Pancreatic lipomatosis is a radiological feature of SDS as well as of the very rare Johanson Blizzard Syndrome but other clinical abnormalities (facial and especially urogenital) should help to differentiate the two entities.

Diagnostic imaging plays a mayor role in the evaluation of the pancreas in infants and children. (18)

Ultrasonography represents the less invasive radiological technique for investigating the pancreatic gland. The pancreas, in SDS affected patients, appears of normal size with increased echogenicity of the silhouette. In contrast, the size of the pancreas in patients with cystic fibrosis usually is decreased. However, ultrasonography might be inadequate as the size of the gland cannot be easily measured with accuracy for its hyperechogenicity or for the presence of abdominal meteorism due to malabsorption.

CT and MRI reveal lipomatosis of the pancreas with greater accuracy but CT has the major disadvantage of using ionizing radiation.

$\mathrm{MRI}$ is an imaging method that allows correlation of the tissue specificity of an organ to its radiological representation, especially in the presence of fat, which is easily recognized in $\mathrm{T} 1$ and $\mathrm{T} 2$ weighted sequences. (19)

Therefore, when pancreatic lipomatosis assessed by MRI, or CT, is associated with clinical characteristics which are suggestive of SDS, diagnosis of SDS should be strongly considered.

\section{Genetic analysis}

In 2002, researchers from Toronto cloned the gene responsible for SDS (SBDS) and identified mutations involved in the disease. The gene has been mapped to chromosome $7 q 11$. Molecular diagnostic testing is now available. (5) The SBDS gene mutations have been detected in most $(80 \%)$, but not all patients with SDS. (20) This suggests that SDS is a genetically heterogeneous disorder. The SBDS gene has an adjacent pseudogene (an identical but nonfunctional gene) on 7q11. The majority of mutations are caused by a recombination during meiosis between SBDS and its highly homologous pseudogene, so that some parts of the pseudogene are incorporated into the normalSBDSgene. The altered SBDS gene received non functional parts from the pseudogene, which made it dysfunctional. (5)

The function of the SBDS encoded protein has not been elucidated. The transcript of the normal gene is widely expressed in the pancreas, bone marrow and leucocytes. Hence, it has been postulated that the SDS phenotype may result from a defect in RNA process- ing that disrupts a cellular function necessaryforhematopoietic, pancreatic and skeletal development. (21)

\section{Acute manifestations and supportive care}

Overwhelming sepsis is a well recognized fatal complication of this disorder, particularly early in life. (2) In case of fever and severe neutropenia, bacterial cultures (from blood, urine, throat) should be performed because of the increased risk of sepsis from the neutrophil migrational defect ob-served in the disease. In addition, patients should receive prompt therapy based on intravenous antibiotics with a broad spectrum coverage until the infection clears up. Granulocyte colony stimulating factor (G-CSF) may be prescribed and patients would probably benefit during acute infections. (2) The most likely bacterial pathogens in SDS are Staphylococcus Aureus, Haemophilus influenzae and Pseudomonas species. (22)

Fatal bleeding, in patients suffering from moderate to severe thrombocytopenia, has been reported. (9) Platelet and/or plasma transfusions and parenteral vitamin $\mathrm{K}$ may be necessary to diminish the risk of bleeding. Although patients with anemia and thrombocytopenia may be supported by red cell and platelet transfusions, very severe neutropenia $(\mathrm{ANC}<200 / \mu \mathrm{L})$ remains an adverse prognostic factor as it is associated with a high mortality rate and risk of lifethreatening infections. (23)

\section{Natural history}

Prognosis is uncertain. Since SDS has been described relatively recently, limited data are available regarding follow up in these affected patients. Deathusuallyoccursfrom overwhelming sepsis or malignancies. Alter et al (1997) reported a median survival age older than 35 years for all patients with SDS. For those patients whose course is complicated by aplastic anemia, the median survival age is 24 years, while those patients whose course is complicated by leukemia have a median survival age of 10 years. (24) 


\section{Management}

Management of children with SDS should be multidisciplinary. The child should be primarily under the care of a local general pediatrician. Long term follow up requires collaboration between pediatric gastroenterologists and hematologists. A significant proportion of patients with SDS require pancreatic enzymes. The dosage should be adapted to the severity of the symptoms, such as steatorrhea, abdominal pain and growth parameters.

Depending on the evolution of hematological abnormalities, a full blood count must be performed every 3-6 months or more frequently if symptoms require. An annual bone marrow biopsy has to be performedforsurveillance of theacquisition of cytogenetic abnormalities. (5)

Complications of the disease, such as sepsis or hematological symptomatic abnormalities, might require admission to an Intensive Care Unit.

\section{Conclusion}

Any child with steatorrhea and poor weight gain, after excluding cystic fibrosis, should be investigated for exocrine pancreatic insufficiency. The assessment of pancreatic dysfunction or the less invasive radiological demonstration of fatty replacement has primary importance. Diagnostic criteria should help in establishing the diagnosis. Other clinical abnormalities support the suspected condition. A specific diag-nostic test for SDS is not available and definitive diagnosis still represents a challenge. Genetic analysis might be the future direction. Clinicians should take into account that life-threatening events may be the first clinical manifestation of the disease.

\section{REFERENCES}

1. Shwachman H, Diamond LK, Oski FA, Khaw KT. The syndrome of pancreatic insufficiency and bone marrow dysfunction. J Pediatr 1964;65:645-63.

2. Dror Y, Freedman MH. Shwachman Diamond Syndrome. A review. Br J Haematol 2002;118:701-13.

3. Smith OP. Shwachman Diamond syndrome. Semin Hematol 2002 Apr;39(2):95-102.

4. Ginzberg H, Shin J, Ellis L, Morrison J, Dror Y, Ip W, et al. Shwachman Syndrome: phenotypic manifestations of sibling sets and isolated cases in a large patient cohort are similar. J Pediatr 1999;135:81-8.

5. Hall GW, Dale P, Dodge JA. Shwachman Diamond syndrome: UK perspective. Arch Dis Child 2006;91:512-24

6. Smith OP, Hann IM, Chessells JM, Reeves BR, Milla P. Haematological abnormalities in Shwachman Diamond syndrome. Br J Haematol 1996;94:279-84.

7. Dror Y, Freedman MH. Shwachman Diamond syndrome: an inherited preleukemic bone marrow failure disorder with aberrant hematopoietic progenitors and faulty microenvironment. Blood 1999;94:3048-54.

8. Mack DR, Forstner GG, Wilshcanski M, Freedman MH, Durie PR. Shwachman syndrome: exocrine pancreatic dysfunction and variable phenotypic expression. Gastroenterology 1996;111:1593-602.

9. Maserati E, Minelli A, Olivieri C, Bonvini L, Marchi A, Bozzola M, et al. Isochromosome 7(q10) in Shwachman Diamond syndrome without MDS/AML and role of chromosome 7 anomalies in myeloproliferative disorders. Cancer Genet Cytogenet 2000;121:167-71.

10. Cunningham J, Sales M, Pearce A, Howard J, Stallings R, Telford N, et al. Does isochromosome 7q mandate bone marrow transplant in children with Shwachman Diamond syndrome? Br J Haematol 2002;119:1062-69.

11. Matikie O, Ellis L, Durie PR, Morrison JA, Sochett EB, Rommens JM, et al. Skeletal phenotype in patient with Shwachman Diamond syndrome and mutations in SBDS. Clin Genet 2004;65:101-12.

12. Aggett PJ, Cavanagh NPC, Matthew DJ, et al. Shwachman Diamond syndrome. A review of 21 cases. Arch Dis Child 1980;55:331-47.

13. Dror Y, Ginzber H, Dalal I, et al. Immune function in patient with Shwachman Diamond syndrome. Br J Hemtol 2001;114:712-17.

14. van Winkelhoff AJ, Schouten-van Meeteren AY, Baart JA, Vandenbroucke-Grauls CM. Microbiology of destructive periodontal disease in adolescent patients with congenital neutropenia. A report of 3 cases. J Clin Periodontol 2000;27:793-98.

15. D'Angio CT, Loyd JK. Nephrocalcinosis in Shwachman's syndrome. Arch Dis Child 1998;64:614-15.

16. Kerr EN. Psychological characteristics in SDS. $2^{\text {nd }}$ International Congress on Shwachman Diamond syndrome. Toronto, Canada: June 2003.

17. Vaughn DD, Jabra AA, Fishman EK. Pancreatic disease in children and young adults: evaluation with CT. Radiographics 1998 SepOct;18(5):1171-87.

18. Nijs E, Callahan MJ, Taylor GA. Disorder of the pediatric pancreas: imaging feature. Pediatr Radiol. 2005 Apr;35(4):358-73.

19. Ruggiero A, Molinari F, Coccia P, Attinà G, Maurizi P, Riccardi R, et al. MRI findings in Shwachman Diamond Syndrome. Pediatr Blood Cancer 2007;00:1-3.

20. Woloszynek R, Rothbaum RJ, Rawls AS, et al. mutation of the SBDS gene is presented in most patients with Shwachman Diamond syndrome. Blood 2004; 104:3588-3590.

21. Federman N, Sakamoto KM. The genetic basis of bone marrow failure syndromes in children. Mol. Genet and Metabol 2005;86:100-9.

22. Grinspan ZM, Pikora CA. Infections in patients with Shwachman Diamond Syndrome. The Pediatric Infectious Disease Journal 2005;4:179-81.

23. Bagby GC, Lipton JM, Sloand EM, Shiffer CA. Marrow failure. Hematology 2004;318-334.

24. Alter PB, Young NS. Shwachman Diamond syndrome. In: Nathan, Oski, editors. Haematology of infancy and childhood. $5^{\text {th }}$ ed. Philadelphia: WB Saunders; 1997;1:276-78. 